\title{
The Different Environmental Dependencies and Evolutions of Giant and Dwarf Galaxies
}

\author{
C.P. Haines ${ }^{1}$, A. Gargiulo ${ }^{1}$, A. Mercurio ${ }^{1}$, P. Merluzzi ${ }^{1}$, \\ F. La Barbera ${ }^{1}$, G. Busarello ${ }^{1}$, and M. Capaccioli ${ }^{1}$ \\ ${ }^{1}$ Osservatorio Astronomico di Capodimonte, via Moiariello 16, I-80131 Napoli, Italy \\ email: chris@na.astro.it
}

\begin{abstract}
The star-formation histories, masses and structural properties of galaxies are strongly dependent on their environment: massive, passively-evolving spheroids dominate cluster cores, while in field regions, galaxies are typically low-mass, star-forming and disk-dominated (e.g. Blanton et al. 2005). Equally the global properties of galaxies have been found to be bimodally distributed around a stellar mass of $\sim 3 \times 10^{10} \mathrm{M}_{\odot}\left(\sim \mathrm{M}^{*}+1\right)$ (e.g. Kauffmann et al. 2003). Largescale spectroscopic surveys have shown that the evolution of massive galaxies is primarily driven by their merger history, rather than through direct interection with the cluster environment, as demonstrated by the finding of massive passively-evolving galaxies in all environments, mild observed environmental trends for their mean stellar ages, and the gradual SF-density relation in which star-formation is still seen to be suppressed in galaxies 3-4 virial radii from the nearest cluster. In contrast, in the dwarf regime $\left(>\mathrm{M}^{*}+2\right)$ dramatic changes are seen in both the luminosity function and red galaxy fraction between those galaxies in the cores of clusters and those at the virial radius (Mercurio et al. 2006, Haines et al. 2006a).

We have examined the origins of the bimodality observed in the global properties of galaxies by comparing the environmental dependencies of star-formation for giant and dwarf galaxy populations. Using SDSS DR4 spectroscopic data to create a volume-limited sample complete to $\mathrm{M}^{*}+3$, we find that the environmental dependences of giant and dwarf galaxies are quite different, implying fundamental differences in their evolution (Haines et al. 2006b). Whereas the star-formation histories of giant galaxies are determined primarily by their merger history, this is not the case for dwarf galaxies. In particular, we find that old or passive dwarf galaxies are ONLY found as satellites within massive halos (clusters, groups or giant galaxies), with none in the lowest density regions. This implies that star-formation in dwarf galaxies must be much more resilient to the effects of mergers, and that the evolution of dwarf galaxies is primarily driven by the mass of their host halo, through effects such as suffocation, ram-pressure stripping or galaxy harassment.
\end{abstract}

Keywords. galaxies:evolution, galaxies:stellar content

\section{References}

Blanton, M.R., Eisenstein, D., Hogg, D.W., Schlegel, D.J. \& Brinkmann, J. 2005, ApJ 629, 143. Haines, C.P., Merluzzi, P., Mercurio, A., Gargiulo, A., Krusanova, N., Busarello, G., La Barbera, F. \& Capaccioli, M. 2006, MNRAS 371, 55.

Haines, C.P., La Barbera F., Mercurio, A., Merluzzi, P. \& Busarello, G. 2006, ApJ (Letters) $647,21$.

Kauffmann G., Heckman, T.M., White, S.D.M., et al. 2003, MNRAS 341, 33.

Mercurio, A., Merluzzi, P., Haines, C.P., Gargiulo, A., Krusanova, N., Busarello, G., La Barbera, F. \& Capaccioli, M. 2006, MNRAS 368, 109. 